\title{
Stroke prevention in atrial fibrillation: findlings from Tuscan FADOI Stroke Registry
}

\author{
Luca Masotti, ${ }^{1}$ Rinaldo Innocenti, ${ }^{2}$ Stefano Spolveri, ${ }^{3}$ Irene Chiti, ${ }^{4}$ Grazia Panigada, ${ }^{4}$ Cristiana Seravalle, ${ }^{5}$ \\ Giancarlo Landini, ${ }^{5}$ Alberto Fortini, ${ }^{6}$ Rino Migliacci, ${ }^{7}$ Raffaele Laureano, ${ }^{8}$ for Tuscan FADOI Stroke Registry In- \\ vestigators
}

${ }^{1}$ UO Medicina Interna, Ospedale di Cecina (LI); ${ }^{2} \mathrm{UO}$ Medicina Interna, Ospedale Careggi, Firenze; ${ }^{3}$ UO Medicina Interna, Ospedale Borgo San Lorenzo, Firenze; ${ }^{4}$ UO Medicina Interna, Ospedale di Pescia (PT); ${ }^{5}$ O Medicina Interna, Ospedale Santa Maria Nuova, Firenze; ${ }^{6}$ UO Medicina Interna, Ospedale San Giovanni di Dio, Firenze; ${ }^{7}$ UO Medicina Interna, Ospedale di Cortona (AR); ${ }^{8} \mathrm{UO}$ Medicina Interna, Ospedale Santa Maria Annunziata, Firenze, Italy

\begin{abstract}
Despite vitamin K antagonists (VKAs) are considered the first choice treatment for stroke prevention in atrial fibrillation $(\mathrm{AF})$, literature shows their underuse in this context. Since data about VKAs use prior and after acute stroke lack, the aim of this study was to focus on management of anticoagulation with VKAs in this context. Data were retrieved from Tuscan FADOI Stroke Registry, an online data bank aimed to report on characteristics of stroke patients consecutively admitted in Internal Medicine wards in 2010 and 2011. In this period 819 patients with mean age $76.5 \pm 12.3$ years were enrolled. Data on etiology were available for 715 of them (88.1\%), 87\% being ischemic and $13 \%$ hemorrhagic strokes. AF was present in 238 patients (33\%), 165 (69.3\%) having a known AF before hospitalization, whereas 73 patients (31.7\%) received a new diagnosis of AF. A percentage of $89 \%$ of strokes in patients with known AF were ischemic and $11 \%$ hemorrhagic. A percentage of $86.7 \%$ of patients with known AF had a CHADS $2 \geq 2$, but only $28.3 \%$ were on VKAs before hospitalization. A percentage of $78.8 \%$ of patients treated with VKAs before stroke had an international normalized ratio (INR) $\leq 2.0 ; 68.7 \%$ of patients with VKAs-related hemorrhagic strokes had INR $\leq 3.0$. Combined endpoint mortality or severe disability in patients with ischemic stroke associated with AF was present in 47\%, while it was present in $19.30 \%$ and $19.20 \%$ of atherothrombotic and lacunar strokes, respectively. At hospital discharge, VKAs were prescribed in $25.9 \%$ of AF related ischemic stroke patients. AF related strokes are burdened by severe outcome but VKAs are dramatically underused in patients with AF, even in higher risk patients. Efforts to improve anticoagulation in this stroke subtype are warranted.
\end{abstract}

\section{Introduction}

Atrial fibrillation (AF) is the widest cardiac arrhythmia in general and hospital populations and it is

Correspondence: Luca Masotti, UO Medicina Interna, Ospedale Santa Maria Nuova, Piazza Santa Maria Nuova 1, 50123 Firenze, Italy. E-mail: luca.masotti@tin.it

Key words: Atrial fibrillation; stroke; vitamin K antagonists; oral anticoagulant therapy; outcome; prevention.

See online Appendix for Members of Tuscan FADOI Stroke Registry Investigators.

Received for publication: 20 November 2013.

Revision received: 13 January 2014.

Accepted for publication: 30 January 2014.

This work is licensed under a Creative Commons Attribution NonCommercial 3.0 License (CC BY-NC 3.0).

CCopyright L. Masotti et al., 2015

Licensee PAGEPress, Italy

Italian Journal of Medicine 2015; 9:134-140

doi:10.4081/itjm.2014.450 an actual major public health problem and maybe it will be much more in the next future. ${ }^{1}$ Prevalence of AF in fact is steadily increasing, especially in the elderly. ${ }^{2-4}$ Up to now, around $1 \%$ of the American general population suffers from $\mathrm{AF}$, but this prevalence reaches around $10 \%$ in the octogenarians, ${ }^{5}$ whereas in Europe the prevalence is esteemed around $5.5 \%$ of general population and $17.8 \%$ in patients over 85 years. ${ }^{6}$ This prevalence could be underestimated due to unrecognized magnitude of silent AF. The risk of stroke in AF patients is from two to seven fold greater compared with patients without $\mathrm{AF}$, mean stroke rate in $\mathrm{AF}$ being around $5 \%$ per year. ${ }^{1}$

The annual risk of stroke is predictable by using practical scores such as $\mathrm{CHADS}_{2}$ and the most recent $\mathrm{CHA}_{2} \mathrm{DS}_{2}$-VASC. ${ }^{1}$ A score $\geq 2$ identifies high stroke risk patients, over $4 \%$ per year for the $\mathrm{CHADS}_{2}$ and over $2.2 \%$ per year for the $\mathrm{CHA}_{2} \mathrm{DS}_{2}$-VASC. ${ }^{1}$

AF related strokes are burdened by higher early and long term mortality, higher rate of complications such as hemorrhagic transformation and severe residual disability compared to the other ischemic stroke subtypes such as the atherothrombotic and lacunar forms. ${ }^{7,8}$

Oral anticoagulant therapy (OAT) with vitamin K antagonists (VKAs) has represented, until the publica- 
tion of results of phase III trials on new oral anticoagulants direct inhibitors of thrombin or activated factor $X,{ }^{9-11}$ the first choice treatment for the stroke prevention in high and moderate risk non-valvular AF patients. ${ }^{1,12,13}$ A meta-analysis had in fact demonstrated that VKAs reduce the relative risk of stroke by $64 \%$ compared to placebo and 39\% compared to aspirin (ASA) despite a risk of intracranial and extracranial bleeding increased by $128 \%$ and $70 \%$ compared to placebo, respectively. ${ }^{14}$ In high and moderate stroke risk patients unsuitable for VKAs due to contraindications or refusals, clopidogrel combined with ASA was considered the first alternative to oral anticoagulants as demonstrated from ACTIVEA study. ${ }^{12,15}$ Before the publication of the ACTIVE-A study, ASA alone was considered a possible alternative to VKAs in patients at high or moderate risk of stroke and was considered the first choice in low risk patients when physicians made decision to treat this group of patients. ${ }^{12}$ However the abovementioned meta-analysis demonstrated that ASA alone gives a non-significant prevention compared to placebo with a relative risk reduction of $19 \%$ [confidence interval (CI) $95 \%$ : from -1 to $35 \%]^{14}$

Despite VKAs are available for the clinical use since fifty years, these drugs have many limitation leading to their underuse in clinical practice. VKAs have a slow onset of action, an unpredictable response due to wide genetic variability and multiple food and drugs interactions and a narrow therapeutic window. ${ }^{16}$ Treatment with VKAs needs of routine laboratory monitoring of the international normalized ratio (INR) with patients discomfort and frequent dose adjustment. ${ }^{16}$ VKAs limitations have lead that only around one half of patients are effectively treated by OAT, low percentage of patients (less than $60 \%$ ) who spent their time in therapeutic range [time in therapeutic range (TTR)] and frequent withdrawals. ${ }^{17}$

Major, especially intracranial, and non major bleedings, which occur respectively around in $3 \%$ and $6 \%$ per year in patients taking VKAs, represent the most feared complication of VKAs treatment. ${ }^{18}$ Intracranial bleedings occur in $0.2-1 \%$ per year, but are often devastating. ${ }^{18}$ Mortality rate in VKAs related intracranial bleedings is $10-40 \%$ higher than non VKAs related intracranial bleedings and it reaches $50-80 \%{ }^{19}$ The majority of patients who develops a VKAs-related intracranial bleeding takes VKAs for cardioembolism prevention in $\mathrm{AF} .{ }^{20}$ Despite increased INR values are a major determinant of risk for VKAs-related intracranial bleeding, from $50 \%$ to $90 \%$ of patients with VKAs-related intracranial bleedings present values of the INR in the therapeutic range (2.0-3.0). ${ }^{20}$

Since literature evidence referred to cardioembolism prevention in AF before, during and after the acute phase of stroke other than its clinical and prognostic characteristics and management lacks, the aim of the present study was to focus on this topic in patients admitted in the Internal Medicine wards of Tuscany (Italy).

\section{Materials and Methods}

We analyzed data of patients enrolled in the Tuscan FADOI Stroke Registry, an online database where we included demographic, clinical, pharmacological, instrumental, functional and prognostic characteristics of patients consecutively admitted for acute ischemic and hemorrhagic stroke in the Internal Medicine wards of Tuscany. Some Stroke Units of Tuscany were also enclosed. The enrollment period ranged from July 1, 2010 to December 31, 2011. Within the entire cohort enrolled, we focused on patients with AF both known before hospital admission or diagnosed during hospitalization. We analyzed demographic data, cardiovascular risk factors and co-morbidities, the annual cardioembolic risk by using the $\mathrm{CHADS}_{2}$ score, the antithrombotic prophylaxis used before the stroke, the clinical severity of stroke as measured by the National Institute of Health Stroke Scale (NIHSS), the occurrence of hemorrhagic transformation, the rate of intrahospital mortality, the functional dependence at discharge as measured by the modified Rankin scale (mRS), the secondary cardioembolic prophylaxis at hospital discharge. The data of patients with ischemic stroke associated with AF were compared with those of patients with atherothrombotic, lacunar or uncertain etiology ischemic stroke subtypes according to TOAST criteria on prognostic outcomes, whereas the data of patients with hemorrhagic stroke and AF were compared with those of patients with hemorrhagic stroke without AF.

\section{Results}

In the analyzed period 819 patients, $50.2 \%$ females and $40.8 \%$ males, with mean age $76.5 \pm 12.3$ year, were enrolled in the Tuscan FADOI Stroke Registry. The majority of patients (more than 90\%) were enrolled from July to December 2010. Data on etiology were available for 715 of them (88.1\%), 87\% being ischemic and $13 \%$ hemorrhagic strokes (Table 1). AF was present in 238 patients (33\%). A number of 201 of 238 patients with $\mathrm{AF}(84.4 \%)$ suffered from ischemic strokes, 37 (15.6\%) for hemorrhagic strokes. A number of 165 patients (69.3\%) had a known AF before hospitalization, whereas 73 patients (31.7\%) received a new diagnosis of AF. $89 \%$ of strokes in patients with known AF were ischemic and $11 \%$ hemorrhagic.

A percentage of $86.7 \%$ of patients with known $\mathrm{AF}$ had a $\mathrm{CHADS}_{2}$ score $\geq 2$ (Figure 1), but only $28.3 \%$ were on VKAs before hospitalization (Figure 2). 
Fourty-nine percent of patients with known AF was treated with antiplatelet drugs, while $22.7 \%$ of patients did not receive any antithrombotic prophylaxis. Table 2 shows the prevalence of $\mathrm{CHADS}_{2}$ score variables in patients with known AF and Figure 3 shows the antithrombotic drugs distribution according to $\mathrm{CHADS}_{2}$ score in this population. Any patient with $\mathrm{CHADS}_{2}$ score 0 was under antithrombotic prophylaxis. VKAs use fell from $40 \%$ in patients with CHADS $_{2}$ score 1 to $16.6 \%$ in patients with $\mathrm{CHADS}_{2}$ score 5 , whereas an- tiplatelet drugs increased from $38.6 \%$ in patients with $\mathrm{CHADS}_{2}$ score 1 to $58.4 \%$ in patients with $\mathrm{CHADS}_{2}$ score 5. A percentage of patients between $15.2 \%$ to $38.6 \%$ with known $\mathrm{AF}$ and $\mathrm{CHADS}_{2}$ score 1-5 received any cardioembolic prophylaxis. $78.8 \%$ of patients treated with VKAs before ischemic stroke had INR values $\leq 2.0$, whereas $68.7 \%$ of patients with VKAs-related hemorrhagic strokes had INR values $\leq 3.0$ (Figure 4).

The burden of ischemic strokes was more severe in patients with AF. More than one half of patients with

Table 1. General characteristics and stroke subtypes.

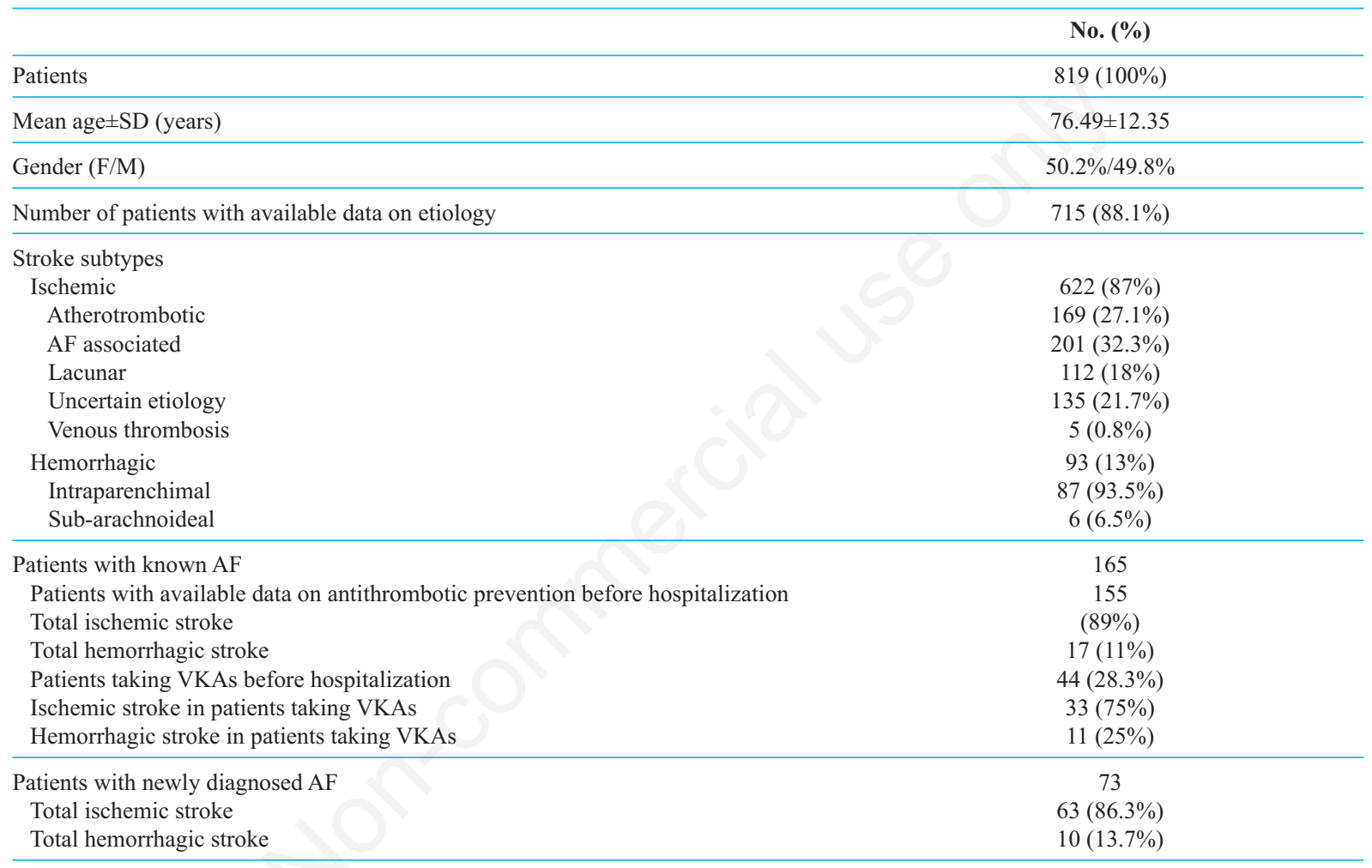

SD, standard deviation; AF, atrial fibrillation; VKAs, vitamin K antagonists.

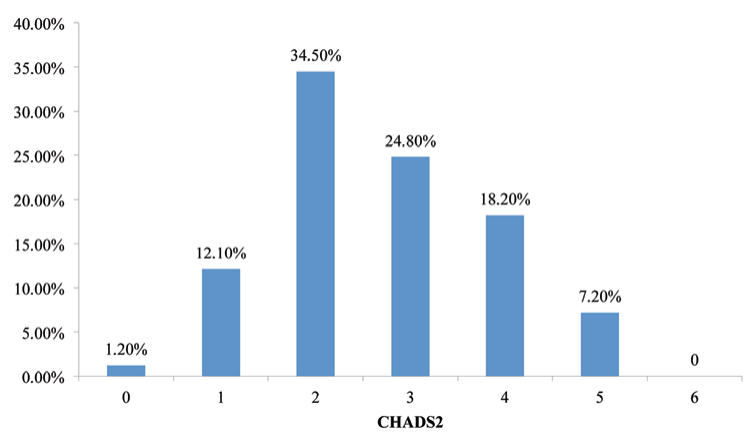

Figure 1. $\mathrm{CHADS}_{2}$ distribution in patients with known atrial fibrillation.



Figure 2. Cardioembolic prophylaxis before hospital admission in patients with known atrial fibrillation. VKAs, vitamin $\mathrm{K}$ antagonists. 
AF-related stroke presented NIHSS score $\geq 10$ points at hospital admission, whereas patients with atherothrombotic or lacunar strokes presented a NIHSS score $\geq 10$ in around $20 \%$ of cases. Hemorrhagic transformation occurred in $12.7 \%$ of patients with AF, while this occurrence was detected in $10.9 \%$ and $2.2 \%$ of atherothrombotic and lacunar strokes, respectively. Mortality in patients with AF-related stroke was near double compared with the other stroke subtypes $(8.8 \%$

Table 2. Prevalence of $\mathrm{CHADS}_{2}$ score variables in patients with known atrial fibrillation.

\begin{tabular}{lc}
\hline Congestive heart failure & $21.6 \%$ \\
\hline Hypertension & $80 \%$ \\
\hline Age $>75$ year & $85.6 \%$ \\
\hline Diabetes & $24.8 \%$ \\
\hline Previous TIA/stroke & $31.2 \%$ \\
\hline
\end{tabular}

TIA, transient ischemic attack.



Figure 3. CHADS ${ }_{2}$ distribution in patients with known atrial fibrillation and cardioembolic prophylaxis according to risk score. VKAs, vitamin $K$ antagonists.

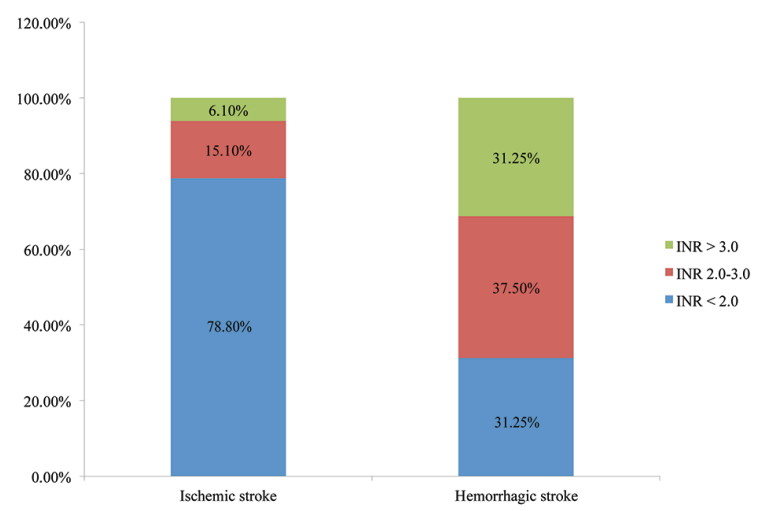

Figure 4. International normalized ratio (INR) values in patients on vitamin $K$ antagonists at hospital admission. in $\mathrm{AF}$ patients $v_{s} 4.7 \%$ and $4.3 \%$ in atherothrombotic and lacunar strokes, respectively). Combined endpoint mortality or severe disability in patients with ischemic stroke associated with AF was present in $47 \%$. Figure 5 summarizes the prognostic endpoints.

In patients with known AF taking VKAs before hospitalization the burden of ischemic stroke was less severe compared with patients with known AF not undergone to this pharmacological treatment. NIHSS score at hospital admission $>10$ points and $\mathrm{mRS} \geq 4$ at hospital discharge were $40 \%$ and $29.6 \%$ respectively in patients treated with VKAs before hospitalization compared to $54 \%$ and $53.1 \%$ in patients untreated. Instead, hemorrhagic transformation was $11.1 \%$ in patients taking VKAs before hospitalization versus $8.1 \%$ in non-taking patients.

At hospital discharge, VKAs were prescribed in $25.9 \%$ of AF-related ischemic stroke patients. The main prescribed antithrombotic treatment was represented by antiplatelet drugs $(61.6 \%)$. None of the patients with VKAs-related hemorrhagic stroke was discharged with VKAs (Figure 6).

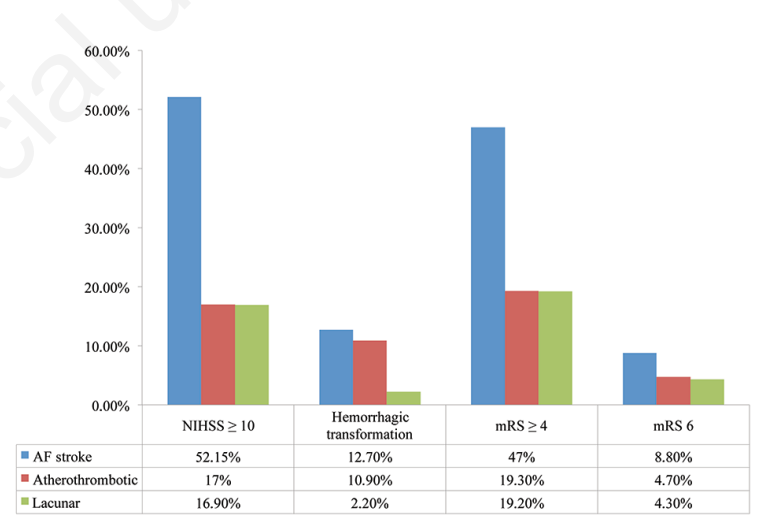

Figure 5. Stroke subtype and outcomes. AF, atrial fibrillation.

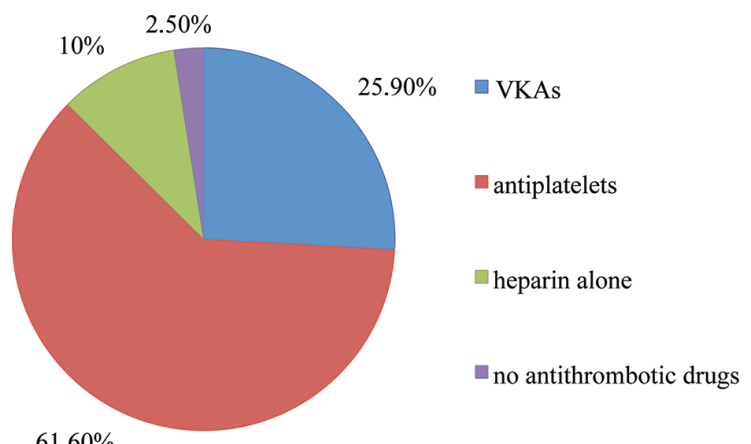

Figure 6. Cardioembolic prophylaxis at hospital discharge. VKAs, vitamin $K$ antagonists. 


\section{Discussion}

Registries or data banks are of utmost importance for understanding and improving the knowledge of many aspects of diseases. The Tuscan Stroke Registry was mainly performed in the second half of 2010 year with the aim of giving, if possible, answers to unresolved questions in the field of stroke patients admitted to internal medicine wards of this Italian region.

In the present study we have focused on AF-related stroke with particular attention to anticoagulation management before and after the acute phase of stroke. The scenario we have found demonstrates the dramatic underuse of VKAs in Tuscany before and after acute stroke even if in high and very high risk patients. Despite population studies show that the VKAs prescription is increased over the years reducing the stroke risk in patients with $\mathrm{AF}$, literature demonstrates that patients with known AF are worldwide yet undertreated, especially when studies focus on preadmission treatment of patients with acute stroke. ${ }^{21-26}$ In this context less than $40 \%$ of patients with known AF are on VKAs treatment. ${ }^{22-26} \mathrm{~A}$ population based study performed in Denmark has demonstrated that the underuse of VKAs is a major determinant of cardioembolic stroke in patient with $\mathrm{AF}$ and could burden for $4-5 \%$ of strokes. ${ }^{27}$

In our study less than one third of patients with known AF admitted to internal medicine wards of Tuscany for acute stroke took VKAs for stroke prevention (28.3\%) with a gap between patients needing VKAs $\left(\mathrm{CHADS}_{2} \geq 2,86.7 \%\right)$ and patients really treated of around $60 \%(58.4 \%)$ and the underuse of VKAs increased in patients with major risk of stroke reaching percentage less than $25 \%$ in patients with $\mathrm{CHADS}_{2}$ score $\geq 4$. Moreover the majority of patients who took VKAs were not protected for stroke risk due to suboptimal anticoagulation control. On the other hand, around one half of patients was treated with antiplatelet drugs, confirming their non-protective effect for stroke prevention in AF, such as found from the abovementioned meta-analyses. ${ }^{14}$ Our findings are not completely surprising, since than an Italian survey in the setting of primary care demonstrated that only around $30 \%$ of patients with AF are treated with VKAs alone $(26.6 \%)$ or in combination with antiplatelet drugs (5.5\%) ${ }^{28}$ Moreover this survey showed that advanced age was a major determinant for not prescribing VKAs and prescribing antiplatelet drugs and only $42 \%$ and $24 \%$ persisted on treatment with VKAs after one and two years from prescription, respectively. ${ }^{28}$

Our study confirms literature reports that burden of ischemic stroke in AF patients is more severe compared to non-AF patients. ${ }^{7}$ In fact, severity at presentation, severe discharge disability and in-hospital mortality were from two to three fold greater in $\mathrm{AF}$ patients compared with non AF patients in our cohort. Of much interest, we found that these outcomes were less severe in patients with known AF taking oral anticoagulants compared to non-taking patients. Our findings confirm those of a much recent study which demonstrated that the use of VKAs before a cardioembolic stroke significantly reduces disability measured by $\mathrm{mRS}$ and mortality compared with no antithrombotic treatment (odds ratio 0.64; CI 95\%: 0.45-0.91). ${ }^{29}$

Another major point of our study is that only around one out of four patients with acute AF-related stroke is discharged from hospital on treatment with VKAs. The much recently published ATA-AF study encompassing more than 7000 patients with $\mathrm{AF}$ and performed in the internal medicine and cardiology wards of Italy, demonstrates that VKAs were prescribed in around one half of patients (49.6\%) discharged from internal medicine wards and internal medicine ward recovery was associated with significantly lower probability to be treated with VKAs. ${ }^{30}$ Only a small proportion of patients (7.1\%) enrolled in internal medicine wards in this study were in the context of acute stroke. ${ }^{30}$ Concerns exist on the optimal timing to start with anticoagulation in the acute phase of stroke. To now, due to literature evidence, available guidelines suggest to avoid anticoagulation in the first $48 \mathrm{~h}$ from cardioembolic stroke onset and to use antiplatelet drugs which have demonstrated the best efficacy/safety profile. ${ }^{31}$ After this time, the choice to anticoagulate or not a patient suffering from acute cardioembolic stroke depends from neurological severity, size of ischemic lesion and cardiac comorbidity. ${ }^{31}$ Briefly, anticoagulation could be started within the first two weeks and preferably within the first week if neurological deficit is mild or moderate, if the size of ischemic lesion is quite small and if there is cardiac comorbidity such as an atrial thrombus or a prosthetic valve. On the other hand anticoagulation should be delayed to the next two weeks from stroke onset if neurological deficit is severe, the size of infarct is wide and there are no cardiac comorbidities. ${ }^{31}$ What are the reasons for the low percentage of patients discharged from hospitals on VKAs treatment in our study are impossible to detect. We could speculate that physicians were reluctant to use VKAs for the fear of bleeding complications in frailty patients or because they found absolute or relative contraindications or poor compliance. Moreover, due to lack of follow-up, we could speculate that in a lot of patients VKAs were started in the next months.

Finally, our study confirms findings of previous studies, highlighting that the majority of patients suffering from spontaneous VKAs-related hemorrhagic stroke are in therapeutic range. ${ }^{20}$ This is a cumbersome problem, since it is widely and wrongly believed that 
the optimal VKAs control could avoid bleeding complications.

\section{Conclusions}

AF-related stroke is a major public health and the lack of adequate and appropriate prevention continue to be disarming as confirmed in our study performed in the Italian medicine wards of Tuscany. Despite VKAs have been considered the first line antithrombotic approach for cardioembolism prevention, these are dramatically underused in clinical practice even in high-risk subjects. Many patients pay the consequence of this under-treatment in terms of ischemic strokes. When VKAs are used, a lot of patients have a sub-optimal treatment control, demonstrated by low TTR and low INR values at hospital admission, consequence of it being the under-protection against cardioembolism. Finally, nevertheless an optimal control of treatment as showed from the good profile of INR values, a not negligible percentage of patients are burdened by spontaneous VKAs-related hemorrhagic strokes.

Therefore efforts to reduce the gap between patients needing cardioembolism prevention and patients really treated or to improve the quality of anticoagulation to avoid often devastating consequences such as ischemic or hemorrhagic stroke are strongly warranted. Much recently new oral anticoagulants have been marketed for stroke prevention in atrial fibrillation due to positive results of phase III randomized clinical trials. ${ }^{9,10}$ Overall, new oral anticoagulants have demonstrated to be effective and safety at least as warfarin, but for many considered endpoints new oral anticoagulants resulted significantly superior to warfarin, especially in safety profile. ${ }^{32}$ Whether new oral anticoagulants could help physicians in improving the gap between patients needing for OAT and patients effectively treated and their outcomes, to date is impossible to predict. However the road is opened.

\section{References}

1. 2012 Focused update of the ESC guidelines for the management of atrial fibrillation. An update of 2010 ESC guidelines for the management of atrial fibrillation. Eur Hearth J 2012;33:2719-47.

2. Andrew NE, Thrift AG, Cadilhac DA. The prevalence, impact and economic implications of atrial fibrillation in stroke: what progress has been made? Neuroepidemiology 2013;40:227-39.

3. Friberg L, Bergfeldt L. Atrial fibrillation prevalence revisited. J Intern Med 2013 [Epub ahead of print].

4. Go AS. The epidemiology of atrial fibrillation in elderly persons: the tip of the iceberg. Am J Geriatr Cardiol 2005; 14:56-61.

5. Go AS, Hylek EM, Phillips KA, et al. Prevalence of diagnosed atrial fibrillation in adults: national implications for rhythm management and stroke prevention: the AnTicoagulation and Risk Factors in Atrial Fibrillation (ATRIA) study. JAMA 2001;285:2370-5.

6. Heeringa J, van der Kuip DAM, Hofman A, et al. Prevalence, incidence, and lifetime risk of atrial fibrillation: the Rotterdam study. Eur Heart J 2006;27:949-53.

7. Lamassa M, Di Carlo A, Pracucci G, et al. Characteristics, outcome, and care of stroke associated with atrial fibrillation in Europe: data from a multicenter multinational hospital-based registry (The European Community Stroke Project). Stroke 2001;32:392-8.

8. Steger C, Pratter A, Martinek-Bregel M, et al. Stroke patients with atrial fibrillation have a worse prognosis than patients without: data from the Australian Stroke registry. Eur Heart J 2004;25:1734-40.

9. Connolly SJ, Ezekowitz MD, Yusuf S, et al. Dabigatran versus warfarin in patients with atrial fibrillation. N Eng J Med 2009;361:1139-51.

10. Patel MR, Mahaffey KW, Garg J, et al. Rivaroxaban versus warfarin in nonvalvular atrial fibrillation. $\mathrm{N}$ Engl $\mathrm{J}$ Med 2011;365:883-91.

11. Granger CB, Alexander JH, McMurray JJ, et al. Apixaban versus warfarin in patients with atrial fibrillation. $\mathrm{N}$ Engl J Med 2011;365:981-92.

12. The Task Force for the management of atrial fibrillation of the European Sociey of Cardiology (ESC). Guidelines for the management of atrial fibrillation. Eur Hearth J 2010;31:2369-429.

13. You JJ, Singer DE, Howard PA, et al. Antithrombotic therapy for atrial fibrillation: antithrombotic therapy and prevention of thrombosis, $9^{\text {th }}$ ed: American College of Chest Physicians Evidence-Based Clinical Practice Guidelines. Chest 2012;141:e531S-75S.

14. Hart RG, Pearce LA, Aguilare MI. Meta-analysis: antithrombotic therapy to prevent stroke in patients who have nonvalvular atrial fibrillation. Ann Intern Med 2007;146:857-67.

15. Connolly SJ, Eikelboom JW, Ng J, et al. Net clinical benefit of adding clopidogrel to aspirin therapy in patients with atrial fibrillation for whom vitamin K antagonists are unsuitable. Ann Intern Med 2011;155:579-86.

16. Ageno W, Gallus AS, Wittkowsky A, et al. Oral anticoagulant therapy: antithrombotic therapy and prevention of thrombosis, 9th ed: American College of Chest Physicians Evidence-Based Clinical Practice Guidelines. Chest 2012;141:e44S-88S.

17. Ogilvie IM, Newton N, Welner SA, et al. Underuse of oral anticoagulants in atrial fibrillation: a systematic review. Am J Med 2010;123:638-45.

18. Lip GY, Andreotti F, Fauchier L, et al. Bleeding risk assessment and management in atrial fibrillation patients: a position document from the European Heart Rhythm Association, endorsed by the European Society of Cardiology Working Group on Thrombosis. Europace 2011; 13:723-46.

19. Cervera A, Amaro S, Chamorro A. Oral anticoagulantassociated intracerebral hemorrhage. J Neurol 2012;259: 212-24.

20. Dequatre-Ponchelle N, Hénon H, Pasquini M, et al. Vitamin $\mathrm{K}$ antagonists-associated cerebral hemorrhages: what are their characteristics? Stroke 2013;44:350-5.

21. Lakshminarayan K, Solid CA, Collins AJ, et al. Atrial fibrillation and stroke in the general medicare popula- 
tion: a 10-year perspective (1992 to 2002). Stroke 2006;37:1969-74.

22. Gladstone DJ, Bui E, Fang J, et al. Potentially preventable strokes in high-risk patients with atrial fibrillation who are not adequately anticoagulated. Stroke 2009;40: 235-40.

23. Indredavik B, Rohweder G, Lydersen S. Frequency and effect of optimal anticoagulation before onset of ischaemic stroke in patients with known atrial fibrillation. J Intern Med 2005;258:133-44.

24. Yu HC, Tsai YF, Chen MC, Yeh CH. Underuse of antithrombotic therapy caused high incidence of ischemic stroke in patients with atrial fibrillation. Int J Stroke 2012;7:112-7.

25. Burgess C, Ingham T, Woodbridge M, et al. The use of antithrombotics in patients presenting with stroke and atrial fibrillation. Ther Clin Risk Manag 2007;3:491-8.

26. Paciaroni M, Agnelli G, Ageno W, et al. Risk factors for cerebral ischemic events in patients with atrial fibrillation on warfarin for stroke prevention. Atherosclerosis 2010;212:564-6.

27. Olsen TS, Rasmussen BH, Kammersgaard LP, Germer U. Strokes attributable to underuse of warfarin and antiplatelets. J Stroke Cerebrovasc Dis 2005;14:55-7.
28. Mazzaglia G, Filippi A, Alacqua M, et al. A national survey of the management of atrial fibrillation with antithrombotic drugs in Italian primary care. Thromb Haemost 2010;103:968-75.

29. Fang MC, Go AS, Chang Y, et al. Thirty-day mortality after ischemic stroke and intracranial hemorrhage in patients with atrial fibrillation on and off anticoagulants. Stroke 2012;43:1795-9.

30. Di Pasquale G, Mathieu G, Maggioni AP, et al. Current presentation and management of 7148 patients with atrial fibrillation in cardiology and internal medicine hospital centers: the ATA AF study. Int J Cardiol 2013; 167:2895-903.

31. Jauch EC, Saver JL, Adams HP Jr, et al. Guidelines for the early management of patients with acute ischemic stroke: a guideline for healthcare professionals from the American Heart Association/American Stroke Association. Stroke 2013;44:870-947.

32. Masotti L, Di Napoli M, Ageno W, et al. Efficacy and safety of new oral anticoagulants compared with warfarin in cardioembolic prophylaxis of patients with non valvular atrial fibrillation. More lights than shadows. Ital J Med 2012;6:153-69. 